\title{
Erratum to Appendix C of "A review of effect sizes and their confidence intervals, Part I: The Cohen's $d$ family"
}

\author{
Jean-Christophe Goulet-Pelletier ${ }^{\mathrm{a} \bowtie}{ }^{\circledR}$ and Denis Cousineau ${ }^{\mathrm{a}} \odot$ \\ ${ }^{\mathrm{a}}$ University of Ottawa
}

\begin{abstract}
The results of the method for estimating confidence intervals according to Steiger and Fouladi (1997) were not displayed correctly in our published paper (Figure 7 in Appendix C). We correct the figure here. The new figure changes our interpretation of the method to a more favorable appreciation.
\end{abstract} Acting Editor Roland Pfister (Uni-

Keywords $\backsim$ Confidence intervals, Cohen’s $d$, pivoting method. versität Würzburg)

\section{Introduction}

The results of the interval estimation method popularized by Steiger and Fouladi (1997), herein called the pivoting method $^{1}$, were displayed erroneously in Appendix $C$ of our recent paper on effect sizes and corresponding confidence intervals (Goulet-Pelletier \& Cousineau, 2018). This error pertains to Figure 7 of the appendix, which displayed a lower variability for the pivoting method than there really is, which gave the impression that the pivoting method was short in its coverage estimations. The error came from the fact that the lower and upper noncentrality parameters were properly found but were plotted relative to the true Cohen's $d$, instead of the observed Cohen's $d$. This made the error bars smaller than they should have been. We doubled-checked all the other simulations reported in our paper and this mistake only applied to the Steiger and Fouladi bars of Figure 7.

We can see in the corrected figure that the pivoting method produces desirable results when the effect size is small or medium for all sample sizes. In fact, for small sample sizes, the pivoting method shows a tighter coverage with effect of small or medium magnitude compared to the non-central method discussed in the article. However, when the effect size is large, there is a systematic underestimation of the lower bound which does not disappear with increasing sample size.
In Appendix C of the published paper, we considered that the pivoting method would be less appropriate than the noncentral method in most scenarios, and more so as the true effect size increases. In light of the corrected figure, we rectify our position by saying that the pivoting method indeed returns coverage similar to the true spread of $95 \%$ of the simulated mean difference in most cases. However, we note that when examining closely the estimated target quantiles (i.e., .025 and .975 for a $95 \%$ coverage), over and above achieving a miss rate of $95 \%$, the recommended method seems more effective in estimating the desired quantile position on average. We also note that the pivoting method is asymmetric in its coverage: the upper end of the distribution is always missed to a larger extent than the lower end. In contrast, the noncentrality method is slightly more balanced. The asymmetry of the pivoting method makes it a less precise estimator of the population quantile compared to the recommended method.

\section{Authors' note}

We are thankful to Eric Fields who brought this issue to our attention.

\section{References}

Clopper, C. J., \& Pearson, E. S. (1934). The use of confidence or fiducial limits illustrated in the case of the bino-

\footnotetext{
${ }^{1}$ The pivoting method, called the inversion method in Steiger and Fouladi (1997), was first introduced in Clopper and Pearson (1934). Briefly, instead of looking for boundaries within the population where the observation is very unlikely (e.g., having a $2.5 \%$ chance of occurrence on either tail), the method searches for alternate populations that would not predict the given observation with more than $2.5 \%$ probability (one to the left of the observation, the other to the right).
} 
Figure 1 \& Figure 7 corrected. Comparison of the Steiger and Fouladi (1997) noncentral method (in red, thick error bars), with the noncentral method discussed in this text (in blue, thin error bars), in a between-group design. Sample size $n$ refers to the number of observations within each group.
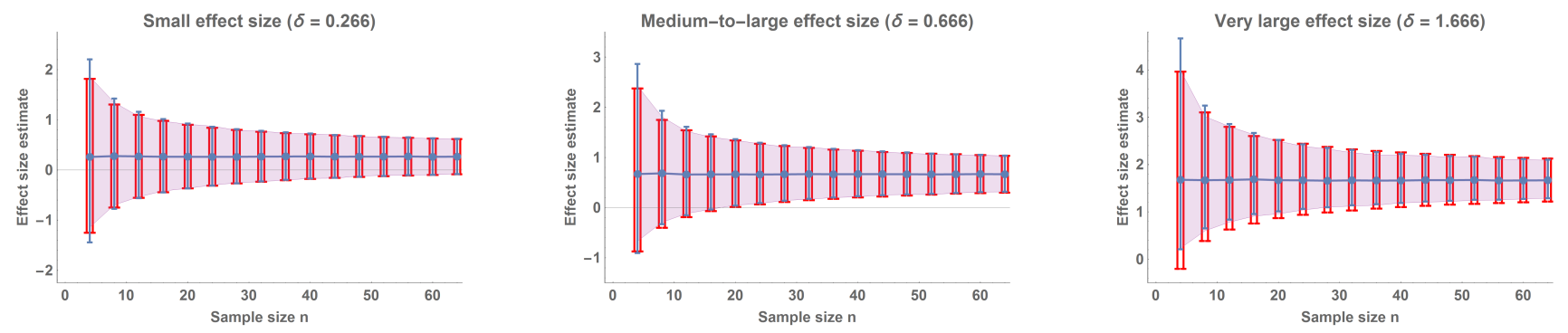

mial. Biometrika, 26(4), 404-413. doi:10.1093/biomet/ 26.4.404

Goulet-Pelletier, J.-C., \& Cousineau, D. (2018). A review of effect sizes and their confidence intervals, part i: The cohen's d family. The Quantitative Methods for Psychology, 14, 242-265. doi:10.20982/tqmp.14.4.p242
Steiger, J. H., \& Fouladi, R. T. (1997). Noncentrality interval estimation and the evaluation of statistical models. What if there were no significance tests? Mahwah, NJ: Erlbaum.

\section{Citation}

Goulet-Pelletier, J.-C., \& Cousineau, D. (2020). Erratum to Appendix C of "A review of effect sizes and their confidence intervals, Part I: The Cohen's d family". The Quantitative Methods for Psychology, 16(4), 422-423. doi:10.20982/tqmp. 16.4.p422

Copyright (C) 2020, Goulet-Pelletier and Cousineau. This is an open-access article distributed under the terms of the Creative Commons Attribution License (CC BY). The use, distribution or reproduction in other forums is permitted, provided the original author(s) or licensor are credited and that the original publication in this journal is cited, in accordance with accepted academic practice. No use, distribution or reproduction is permitted which does not comply with these terms.

Received: 24/04/2020 Accepted: 28/04/2020 